\title{
Economics of DC wind collection grid as affected by cost of key components
}

\author{
Georgios Stamatiou, ${ }^{1, *}$, Kailash Srivastava ${ }^{2}$, Muhamad Reza $^{2}$, Pericle Zanchetta ${ }^{1}$ \\ ${ }^{1}$ University of Nottingham, Nottingham, UK \\ ${ }^{2}$ ABB Corporate Research, Västerås, Sweden \\ * Corresponding author. +30 6976276783, E-mail: geostamatiou@gmail.com
}

\begin{abstract}
Using High Voltage Direct Current (HVDC) transmission lines to connect offshore wind parks to the onshore power grid has been proven technically advantageous to AC solutions and more cost-effective for relatively long transmission distances $(>70 \mathrm{~km})$. The concept of applying DC technology can be expanded to the collection grid. DC collection grid offshore wind parks can be developed only if several key components currently nonexistent are available. The technical challenges involved can result in the unpredictability of their costs. This paper investigates the effect the uncertainty of the key components' cost can have on the overall economic performance of DC collection grid offshore wind parks. Results for a wide cost range are presented and corresponding cost boundaries which secure the economic viability of such parks were determined.
\end{abstract}

Keywords: DC grid, Offshore Wind park, Techno-economics, DC/DC.

\section{Introduction}

In the future the size of offshore wind farms and the distance to shore are expected to increase, thereby leading to higher losses in the AC collection grid and AC transmission. DC technologies can provide lower losses and use cheaper cables than their AC counterparts, thereby compensating for the increased cost of the necessary power electronic devices. HVDC transmission systems have already been proven technically advantageous and cost-effective over AC transmission for distances longer than 60-70 km according to [1], and applying DC technologies not only to the transmission system but also to the collection grid of offshore wind parks could prove additionally effective according to [2].

Key components refer to those components that are not at present available in the market but are needed to realize DC collection grids. According to [3], the following components are identified as key components: DC Circuit Breaker and DC/DC Converter which includes the Medium Frequency Transformer. DC breakers for high power applications have not been constructed and DC/DC converters are not currently available for high power rating beyond 1 or 2 MW [6]. Lundberg in [10] conducts a techno-economic analysis on DC wind parks but omits the cost of DC switchgear and underestimates the cost range of the DC/DC converters, neglecting their challenging nature.

This paper investigates the effect of cost uncertainties of key components on the economic viability of offshore DC wind collection grid. Four different wind park layouts are considered. Initial assumptions on the key components' costs are made and using a cost-benefit programming tool, cost evaluation scenarios take place by altering the cost of the key components. Finally, cost boundaries are defined, beyond which any DC collection grid layout is not economically competitive.

\section{Key components}

\subsection{Circuit breaker}

Realising DC collection grid for offshore wind farms requires the existence of switching and protection devices specifically designed for DC grids. DC Circuit breakers are a characteristic example. Figure 1 (a) presents a typical DC grid. A capacitor is required to stabilize the 
voltage and reduce the voltage ripple at the DC side of the converter. Due to this capacitor, the system can be described by an equivalent constant voltage source $\mathrm{V}_{\text {Grid }}$ and grid impedance $\mathrm{L}_{\text {Grid }}$ as in Figure 1 (b). When a short-circuit occurs, all of the grid voltage is applied across the equivalent resistance. As a result, there is a large and constant voltage across the inductance, leading to a constant and steep rate of rise of the current. Therefore, the fault current reaches dangerously high values quite quickly. Decreasing the rate of rise of the current implies the forced application of voltage across the circuit breaker so that the voltage drop across the inductance is decreased. Additionally in contrast to an AC system, a DC system by definition does not have current zero crossings, which could aid for the safe interruption of the fault current.

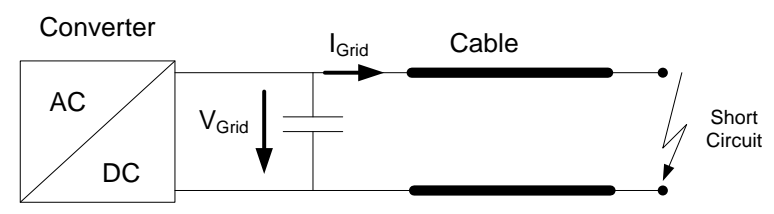

(a)

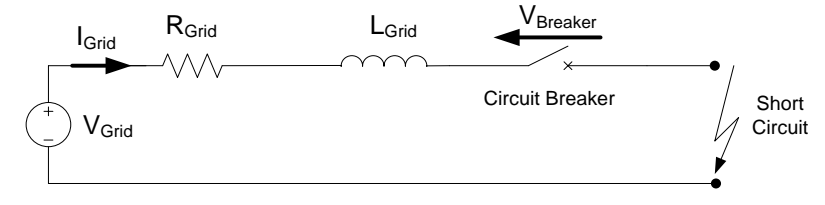

(b)

Figure 1. a) Representation of DC grid under short circuit condition b) Equivalent circuit of a DC grid under short circuit conditions

Therefore a high-power DC breaker must a) be able to act very rapidly to avoid extremely high currents and b) Allow active turn-off.

\subsection{DC/DC Converter}

The requirement for transforming voltages in a high-power DC grid is one of the major challenges towards the realization of DC power networks including DC collection grid. Different topologies for DC/DC converters have been developed using medium or highfrequency AC link as discussed in [4], based on the general model of Figure 2. A galvanic isolated DC/DC converter consists of an inverter at the input side, an AC link consisting of a transformer and an inverter at the output side to transform back to DC. The voltage at the endpoints of the transformer does not need to be sinusoidal or at $50-60 \mathrm{~Hz}$. Hence the frequency of the alternating voltage can be equal to the switching frequency and a rectangular waveform of such frequency can be applied to the transformer. The AC link is based on a medium/high frequency transformer, resulting in galvanic isolation which is important for security reasons in high power applications.
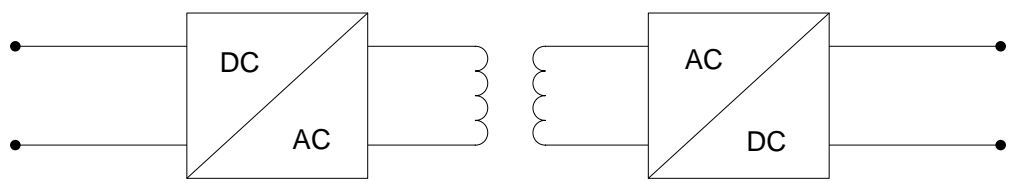

Figure 2. General topology of a galvanic isolated DC/DC converter

The overall dimensions of such a transformer are significantly reduced due to the higher operating frequency, resulting in a compact DC/DC converter. The transformer can be single or three phase. The transformer can be single or three phase with the latter being more advantageous for high-power applications [5]. 


\subsection{Medium Frequency Transformer}

As mentioned in section 2.2, the fundamental component of a high power DC/DC converter is the medium frequency transformer, which is also the most challenging to construct for high power applications. The non-sinusoidal excitation of the core and the use of high-frequency components require sophisticated approach methods. The material and the design of the core of the transformer are of utmost importance. Traditional low frequency-high voltage-high power transformers use laminated cores. However, this concept cannot be applied to medium frequency transformers. Increasing the frequency leads to excessive losses and the insulation between the core sheets will lead to a reduced thermal conductivity of the core [3].

\section{Methodology}

\subsection{Layouts}

For the scope of this paper, four different offshore wind park layouts where investigated: "Small DC", "Large DC", "Series DC" and "AC/DC". These designs are thoroughly presented in [10] and [7]. All of them have a DC transmission link and DC collection grid with the notable exception of the AC/DC which has an AC collection grid. AC is currently the only technology used in the collection grid of wind parks and therefore, AC/DC layout is used as a benchmark for the rest fully DC wind park designs. It is important to mention that the turbines in all DC collection grids, the turbines consist of a Permanent magnet generator coupled to a rectifier and then to a DC/DC converter while the turbines of the AC/DC park consist of a Doubly Fed Induction Generator (DFIG) connected to a transformer.

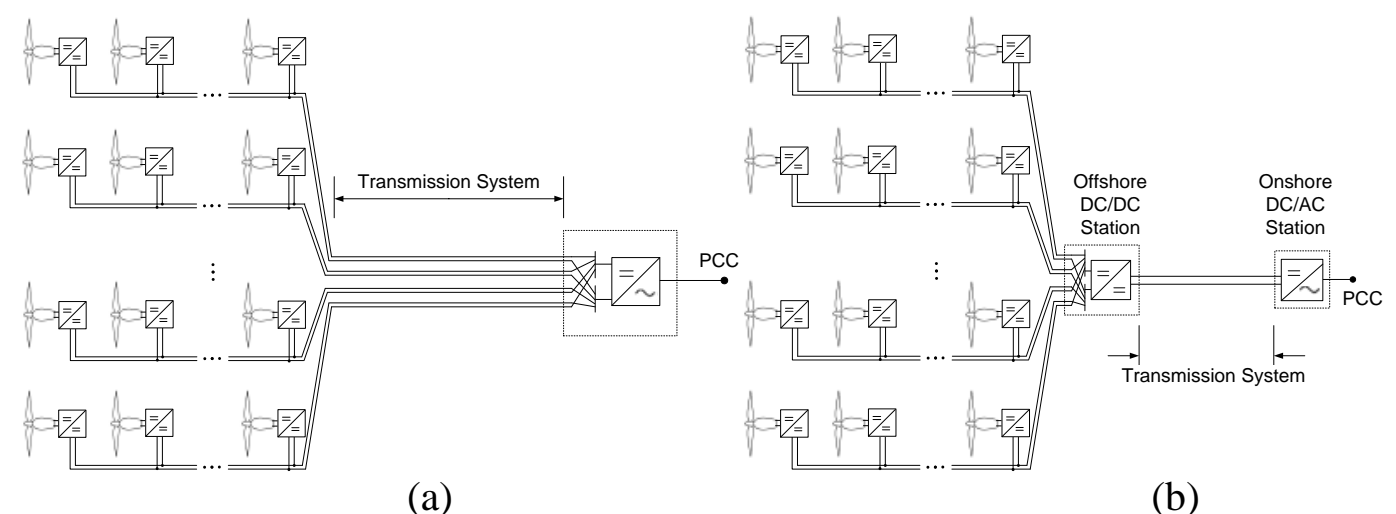

(a)

(b)

Figure 3. a) Small DC offshore wind park b) Large DC offshore wind park

The small DC layout presented in Figure 3 (a) has DC feeders of Medium Voltage (MV) which are extended to the onshore inverter, located just before the Point of Common Coupling (PCC) to the main grid. Figure 3 (b) presents the Large DC layout which resembles the Small DC but uses a large offshore DC/DC converter on a platform, to achieve High Voltage DC (HVDC) on the transmission system, resulting in decreased transmission losses. The Series DC layout is featured in Figure 4 (a). The turbines have a MV DC output and are connected in series, resulting in HVDC along each feeder and also on the transmission line, without the need of an additional offshore DC/DC converter. The AC/DC layout is depicted in Figure 4 (b), having a conventional AC collection grid and an HVDC transmission system. 


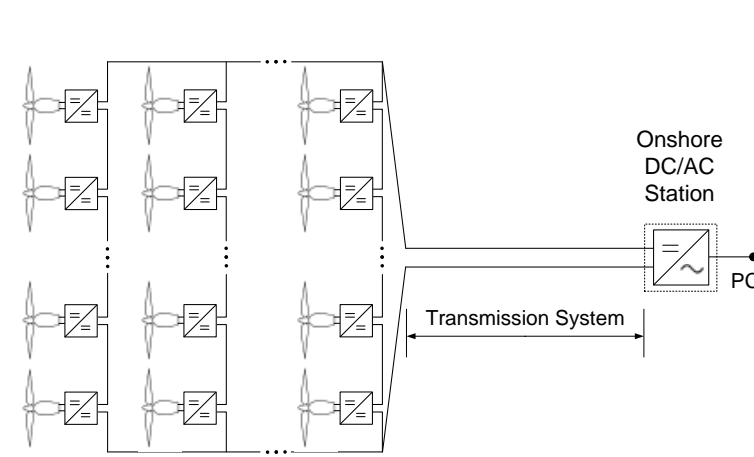

(a)

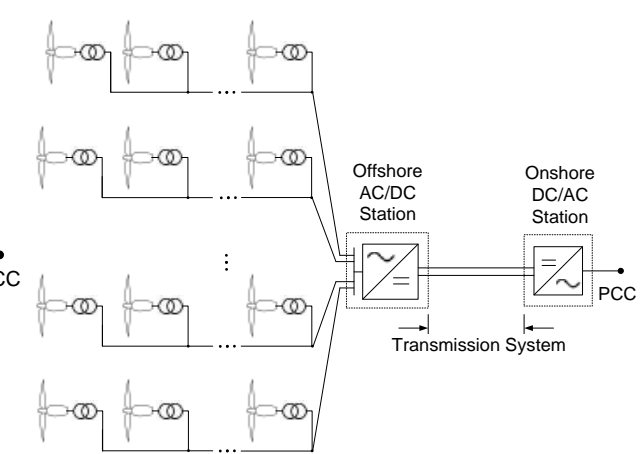

(b)

Figure 4. a) Series DC offshore wind park b) AC/DC offshore wind park

\subsection{Energy Cost}

The minimum cost of energy production for any desired wind farm project is described by the Energy Cost and is given in currency units per kWh delivered to the PCC. Its calculation is given by equation (1):

Energy Cost $=\frac{\text { Total capital cost } \cdot \frac{\mathrm{r}(1+\mathrm{r})^{N}}{(1+\mathrm{r})^{N}-1}+\mathrm{O} \& \mathrm{M}}{\text { Average power production } \cdot \mathrm{T}}$

where O\&M is the total annual Operation and Maintenance costs, $\mathrm{r}$ is the interest rate, $\mathrm{N}$ is the lifetime of the wind farm in years and $\mathrm{T}$ is the average operational hours per year in the form of $\mathrm{T}=24 \cdot 365$ hours $\cdot 0.95$, where the last term expresses a park availability of $95 \%$ annually. Ideally, a project should have the lowest energy cost possible.

\subsection{Cost-Benefit Tool}

A Cost-Benefit programming tool was developed in order to calculate the Energy Cost. It consists of sets of function/script in Matlab environment while the management of input and output information is provided by dedicated Microsoft Office Excel files. Every desired offshore wind park is represented by a dedicated Excel file, containing all the important technical and economical parameters that precisely describe its unique configuration. The tool imports this information and after performing a sequence of calculations, determines the Energy Cost according to equation (1).

\subsubsection{Energy yield Calculation}

The determination of the Energy Cost requires the calculation of the average power production of the wind parks. This is done by considering every wind park as a complex model consisting of interconnected elements. The components which have been modeled are the wind turbines, transformer, DC/DC converter, HVDC station and cables (AC and DC). The modeling details are available in [7]. The power at PCC is calculated for a given instantaneous wind speed and to describe the variations in the wind speed over time, a density function is used and in particular the Weibull distribution $f(w)$ described in [8], were w is the wind speed in $\mathrm{m} / \mathrm{s}$, as the ideal representation of real measured wind data. Using the probability density function, the average power can be generally calculated as:

$P_{\text {average }}=\int_{0}^{\infty} P(w) \cdot f(w) \cdot d w$ 
where $\mathrm{P}(\mathrm{w})$ is the power at PCC for wind speed of $\mathrm{w} \mathrm{m} / \mathrm{s}$.

\subsubsection{Capital and maintenance costs}

Wind park related cost data was obtained from published sources. A lot of information was available in [9] and [10]. The gathered data is referred in [7] regarded the following: Wind turbine (different for AC and DC grid), foundations, transformer, HVDC stations, cables (AC and DC), cable installation, offshore platform, DC/DC converters (according to type of usage) and switchgear (for $\mathrm{AC}$ ). No resource was found on DC switchgear and due to the challenging construction, we assumed that setting its cost as double the AC switchgear. Additionally, Lundberg in [10] claims that since a DC/DC converter contains approximately the same amount of semiconductors as an AC/DC converter of the same rating, it is reasonable to have the same cost as the latter, namely 1 SEK/VA. However, regarding the complexities involved in the construction of DC/DC converters, such a cost was not considered adequate. A more careful and trustworthy approach was adopted. The converters on the turbines of the Series DC layout were considered at 3 SEK/VA owing to the high insulation requirements. The high power converters used in the offshore platform of the Large DC were considered at 2 SEK/VA because of the complex construction and the DC/DC converters on the turbines of the Small and Large DC schemes cost 1.5 SEK/VA due to the complexities involved.

In order to calculate the O\&M costs, the components which require maintenance were gathered in three sets of related components: "Turbine", "Collection grid" and "Transmission system". According to literature sources, O\&M can be taken as a certain percentage of the capital cost. Therefore the corresponding "Turbine O\&M", "Collection grid O\&M" and "Transmission system O\&M" were defined as $4 \%, 2 \%$ and $0.5 \%$ percentages respectively, of the total capital costs of the three initial sets.

\subsection{Simulation considerations and assumptions}

For each of the four suggested layouts, three different power capacities were considered: a) $160 \mathrm{MW}$, b) $500 \mathrm{MW}$ and c) $1200 \mathrm{MW}$. Regarding the design considerations, the collection grid voltage was set at $30 \mathrm{kV}$ DC for DC grids and $30 \mathrm{kV}$ AC for the AC grids. Additionally the HVDC transmission links were rated at $\pm 150 \mathrm{kV}$ for the 160 and $500 \mathrm{MW}$ cases and $\pm 320 \mathrm{kV}$ for the $1200 \mathrm{MW}$. Finally each feeder of the collection grid consisted of 10 turbines in all cases. The design peculiarity of the $1200 \mathrm{MW}$ Series DC requested the need for $32 \mathrm{kV}$ DC at the exit of the turbines' DC/DC converters and 20 turbines per feeder. Finally, every wind park was simulated for transmission distances in the range of $0-200 \mathrm{~km}$. Additionally, several assumptions were made as in Table 1.

Table 1. Simulation assumption

\begin{tabular}{rc}
\hline Parameter & Assumption \\
\hline Wake effect & Neglected \\
Cable inventory & Maximum 4 different cables per model \\
Turbine's power rating & 2MW \\
Turbine power curve & Same for DFIG (before transformer) and FPCG (before DC/DC \\
Weibull shape factor & converter) \\
Converter loss curve & $\mathrm{k}=2$ \\
Interest rate & Same for HVDC and DC/DC \\
Life cycle & $7 \%$ per annum \\
Average wind speed & 25 years \\
& $10 \mathrm{~m} / \mathrm{s}$ for all cases and all transmission distances \\
\hline
\end{tabular}




\section{Results}

\subsection{Cost of DC/DC converters}

In order to determine how sensitive the economic viability of the DC collection grid is with respect to the DC/DC converter cost, all costs were kept unchanged and the DC/DC converter cost was altered. Assuming that the cost proportion between the 3 different kinds of DC/DC converters as described in 3.3.1 remains always fixed, the following concept is followed: all of these values are multiplied by a common percentage representing the cost fluctuation i.e. a $50 \%$ percentage means that the new costs are half the original ones.

Figure 5 presents the results in Energy Cost terms for all layouts of the 160 MW power rating, for percentages of $50 \%, 100 \%$ and $300 \%$. For a specific power capacity, the energy cost curves of the Large DC, Series DC and AC/DC seem to be parallel. This happens because these three layouts share the same transmission lines and increasing the distance will only increase the length of the cables. As a result, all three layouts will experience the same amount of capital and O\&M cost increase and therefore energy cost increase. The order of the energy cost curves is defined by the costs with no transmission line involved. This is the energy cost at $0 \mathrm{~km}$ of distance. The Small DC differs in a great degree because it features multiple transmission cables and for increasing distance, the huge capital and O\&M cost as well as excessive transmission system losses, force the energy cost to increase at a steep rate. As expected, the energy cost curve of the AC/DC layout remains the same in all three scenarios as it contains no DC/DC converters. On the contrary, the energy cost of the Small DC, Large DC and Series DC increase for increasing percentage (more expensive converters). As observed in the figure and also confirmed in [7], the Small DC rarely has the smallest energy cost and when this happens, it is only for distances less than $2-3 \mathrm{~km}$. Additionally, its energy cost increases almost exponentially due to the large number of costly transmission lines and increased losses. Therefore, Small DC is of no interest for offshore development where distances are expected to be in the order of 10 s to $100 \mathrm{~s}$ of $\mathrm{km}$ and as a result, it will not be depicted again in the following figures.
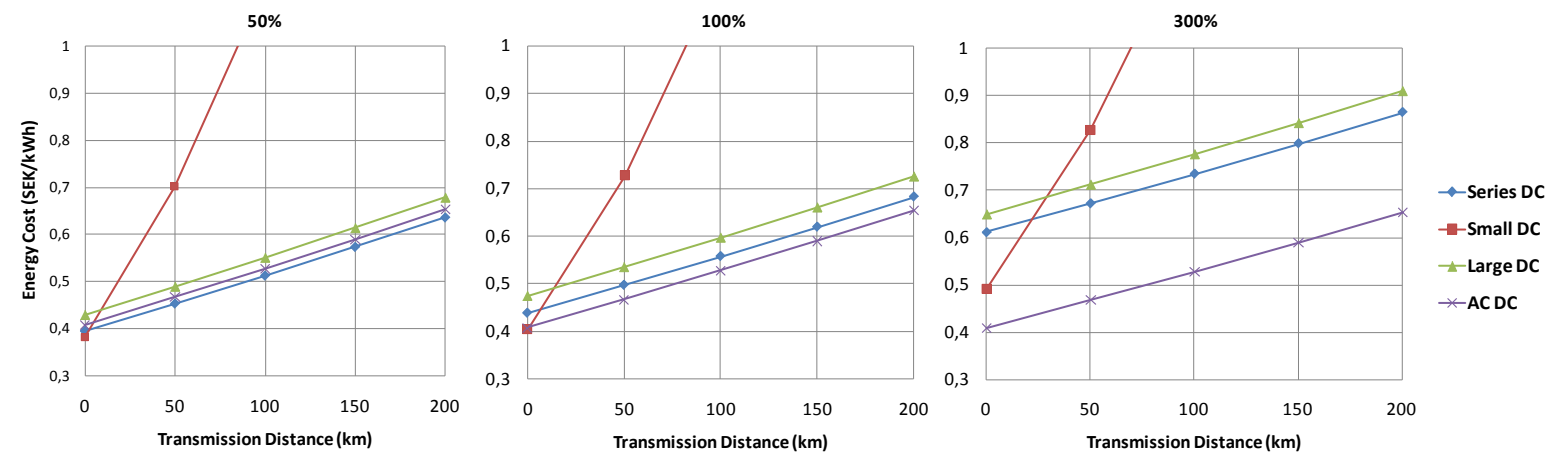

Figure 5. Energy cost curves of 160MW layouts for DC/DC cost percentages of 50\%, 100\% and 300\%

For very small percentages, the Series DC has the smallest energy cost in all distances with the AC/DC following. For increasing percentage, AC/DC remains constant with the Series DC moving upwards until they coincide for a certain percentage. After this, the AC/DC maintains the smallest energy cost for all distances, and effectively all DC collection grid layouts have been ruled out of the competition. In every case, the Large DC has always higher energy cost than both the Series DC and the AC/DC. Therefore, only AC/DC and Series DC are considered as competitive layouts. This break-even percentage is different for each of the three power ratings and the findings are presented in Table 1. 
Table 2. Break-even DC/DC cost percentages for each power rating

\begin{tabular}{cc}
\hline Wind park Power rating & Break-even percentage \\
\hline $160 \mathrm{MW}$ & $67 \%$ \\
$500 \mathrm{MW}$ & $94 \%$ \\
$1200 \mathrm{MW}$ & $145 \%$ \\
\hline
\end{tabular}

If the results from all layouts and capacities are presented in the same graph as in Figure 6, it can be seen that for the basic scenario of $100 \%$ percentage, the AC/DC technology has the lowest energy cost up to a distance of $\sim 100 \mathrm{~km}$, with the Series DC having the lowest energy cost for any greater distance. It was observed that by decreasing the DC/DC converter cost, the AC/DC technology was the most cost-benefit solution for smaller distances until it was eventually not competitive at all, with the Series DC being the cheapest for all distances. Reversely, for increasing converter cost the dominance of the Series DC is shifted to higher distances until a certain point when AC/DC has the lowest energy cost for all distances within the range of $0-200 \mathrm{~km}$.
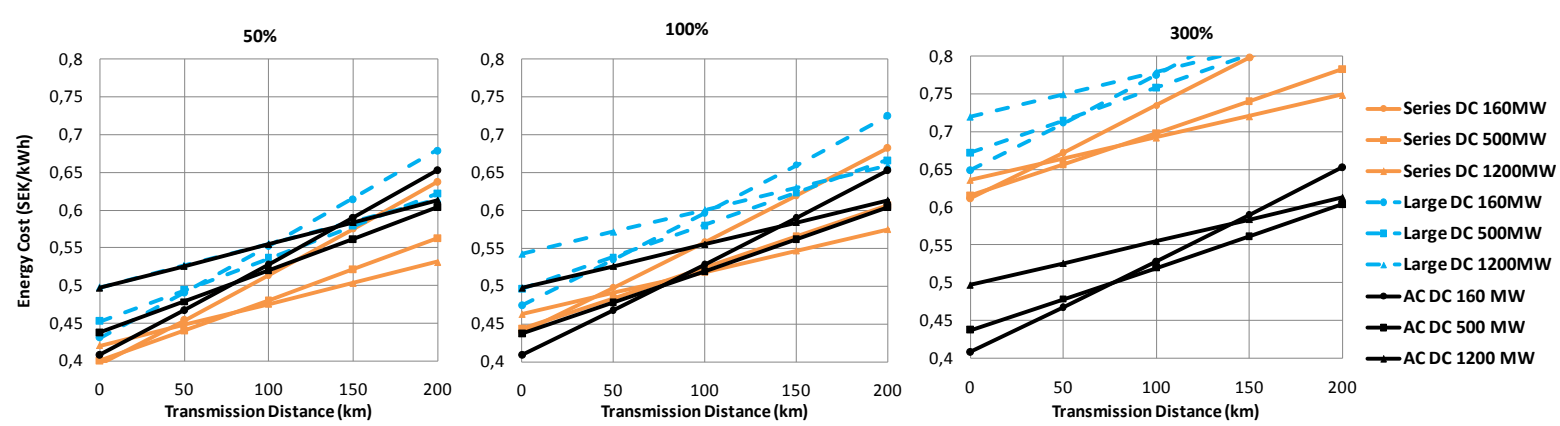

Figure 6. Energy Cost curves of Series DC, Large DC and AC/DC for DC/DC cost percentages of $50 \%, 100 \%$ and $300 \%$.

It was determined that these two percentages are the break-even values for the $160 \mathrm{MW}$ and $1200 \mathrm{MW}$ as in Table 2. Therefore, for a percentage lower than $67 \%$, the Series DC is the technology with the lowest energy cost for all distances, then AC/DC begins to be the cheapest up to certain distances, and when the percentage exceeds $145 \%$, the AC/DC is the cheapest overall with no DC collection grid layout being competitive any more. The general conclusion is that if DC collection grid is to be regarded as a future solution for offshore wind parks for distances up to $200 \mathrm{~km}$, Series DC is the most cost-benefit wise interesting approach and the cost of the DC/DC converters must not exceed 145\% of the originally assumed costs described in Table 1 . When the cost percentage is less than $67 \%$, Series DC (therefore DC collection grid) is the best solution in all distances.

\subsection{DC switchgear cost}

The same concept as before was followed in the case of the DC switchgear cost were the uncertainties in the DC breaker cost are involved. The original cost was multiplied by a percentage and break-even costs were determined as in Table 3. It is interesting to notice that no break-even percentage was evaluated for the $160 \mathrm{MW}$ case, meaning that AC/DC has always the lowest energy cost for all distances, no matter how cheap the DC switchgear gets.

When all the results are compared, the Series DC remains competitive until the percentage regarding the cost of the DC switchgear exceeds $378 \%$ and the AC/DC technology features the lowest energy cost for all $200 \mathrm{~km}$. This margin is high due to the smaller contribution of 
Table 3. Break-even DC switchgear cost percentages for each power rating

\begin{tabular}{cc}
\hline Wind park Power rating & Break-even percentage \\
\hline $160 \mathrm{MW}$ & Nonexistent \\
$500 \mathrm{MW}$ & $54 \%$ \\
$1200 \mathrm{MW}$ & $378 \%$ \\
\hline
\end{tabular}

the DC switchgear to the overall capital costs, compared to the DC/DC converters. Even if the percentage drops to $0 \%$, AC/DC will still be the most competitive for distances up to $60 \mathrm{~km}$.

\section{Conclusions}

Under the circumstances where offshore wind farms are growing in the capacity and distance from shore, DC wind collection grid along with DC transmission can be the most cost effective solution. However, any development of such designs depends on the presence of currently unavailable components, namely DC/DC converters and DC circuit breakers, and also on their expected costs. Specifically, the cost of DC/DC converters must be quite close to the suggested values of this paper while there is a lot more margin for the DC circuit breaker's cost. Any future research aiming to materialise the needed key components must be done with respect to the indicated cost boundaries and general findings of this paper.

\section{References}

[1] P. Bresesti, W. L. Kling, R. L. Hendriks, and R. Vailati, "HVDC connection of offshore wind farms to the trans-mission system," IEEE Trans. Energy Conv., vol. 22, no. 1, pp. 37-43, Mar. 2007

[2] Macken, K.J.P.; Driesen, J.L.J.; Belmans, R.J.M., "A DC Bus System for connecting offshore wind turbines with utility system," European Wind Energy Conference 2001, Copenhagen, Denmark, 2-6 July 2001, pp. 1030-1035.

[3] Christoph Meyer, "Key Components for Future Offshore DC Grids" $\mathrm{PhD}$ thesis submitted to Electrical Engineering Department, RWTH Aachen.

[4] Ranganathan, V.T., Ziogas, P.D. Stefanovic, V.R., “A regulated DC-DC voltage source converter using high frequency link,” IEEE Transaction on Industry Application (IAS) 18 (1982), No. 3, pp. 279-287.

[5] Jacob, J.H.A.M., "Multi-phase Series Resonant DC/DC Converters,” Aachen Germany, RWTH Aachen University, Aachener Beitrage des ISEA Band 42, PhD Thesis 2006.

[6] Engel, B., Victor, M., Bachman, G., Falk, A., “15kV/16.7 Hz energy Supply System with Medium Frequency Transformer and 6.5 kV IGBTs in Resonant Operation,” European Power Electronics Conference (EPE), Toulouse, France, September 2003.

[7] Georgios Stamatiou, "Techno-Economical Analysis of DC Collection Grid for Offshore Wind Parks", MSc. Thesis, University of Nottingham, 2010, http://hermes.eee.nottingham.ac.uk/mcf/Thesis.pdf

[8] http://www.weibull.com/AccelTestWeb/weibull_distribution.htm

[9] B. Van Eeckhout," The economic value of VSC HVDC compared to HVAC for offshore wind farms," Master Thesis submitted to Katholik University of Leuven.

[10] Stefan Lundberg, "Performance comparison of wind park configurations," Chalmers University of Technology, 2003. 